Internat. J. Math. \& Math. Sci.

Vol. 24, No. 9 (2000) 643-647

S0161171200004154

(C) Hindawi Publishing Corp.

\title{
SOME SUFFICIENT CONDITIONS FOR STRONGLY STARLIKENESS
}

\section{MILUTIN OBRADOVIĆ and SHIGEYOSHI OWA}

(Received 22 November 1999)

ABSTRACT. We consider strongly starlikeness of order $\alpha$ of functions $f(z)=z+a_{n+1} z^{n+1}$ $+\cdots$ which are analytic in the unit disc and satisfy the condition of the form

$$
\left|f^{\prime}(z)\left(\frac{z}{f(z)}\right)^{1+\mu}-1\right|<\lambda, \quad 0<\mu<1,0<\lambda<1 .
$$

Keywords and phrases. Analytic function, strongly starlikeness, subordination.

2000 Mathematics Subject Classification. Primary 30C45.

1. Introduction and preliminaries. Let $H$ denote the class of functions analytic in the unit disc $U=\{z:|z|<1\}$ and let $A \subset H$ be the class of normalized analytic functions $f$ in $U$ such that $f(0)=f^{\prime}(0)-1=0$. For $n \geq 1$ we put

$$
A_{n}=\left\{f: f(z)=z+a_{n+1} z^{n+1}+\cdots \text { is analytic in } U\right\}
$$

and $A_{1}=A$.

A function $f \in A$ is said to be strongly starlike of order $\alpha, 0<\alpha \leq 1$, if and only if

$$
\frac{z f^{\prime}(z)}{f(z)} \prec\left(\frac{1+z}{1-z}\right)^{\alpha}
$$

where $\prec$ denotes the usual subordination. We denote this class by $S(\alpha)$. If $\alpha=1$, then $S(1) \equiv S^{*}$ is the well-known class of starlike functions in $U$ (cf. [1]).

In this paper, we find a condition so that $f \in A_{n}$ satisfying

$$
f^{\prime}(z)\left(\frac{z}{f(z)}\right)^{1+\mu} \prec 1+\lambda z, \quad 0<\mu<1,0<\lambda<1,
$$

is in $S(\alpha)$. Also, we consider an integral transformation.

We note that the author in [4] determined the values for $\lambda$ in (1.3) which implies starlikeness in $U$. Recently, Ponnusamy and Singh [5] found the condition which implies the strongly starlikeness of order $\alpha$, but for $\mu<0$ in (1.3). By using the similar method as in [5] we consider strongly starlikeness in the case (1.3).

First, we cite the following lemma.

LEMMA 1.1. Let $Q \in H$ satisfy the subordination condition

$$
Q(z) \prec 1+\lambda_{1} z, \quad Q(0)=1,
$$

where $0<\lambda_{1} \leq 1$. For $0<\alpha \leq 1$, let $p \in H, p(0)=1$ and $p$ satisfy the condition

$$
Q(z) p^{\alpha}(z) \prec 1+\lambda z, \quad 0<\lambda \leq 1 .
$$


Then for

$$
\sin ^{-1} \lambda+\sin ^{-1} \lambda_{1} \leq \frac{\alpha \pi}{2}
$$

we have $\operatorname{Re}\{p(z)\}>0$ in $U$.

This is the special case of the more general lemma given in [5].

2. Results and consequences. For our results we also need the following two lemmas.

LEMMA 2.1. Let $p \in H, p(z)=1+p_{n} z^{n}+\cdots, n \geq 1$, satisfy the condition

$$
p(z)-\frac{1}{\mu} z p^{\prime}(z) \prec 1+\lambda z, \quad 0<\mu<1,0<\lambda \leq 1 .
$$

Then

$$
p(z) \prec 1+\lambda_{1} z
$$

where

$$
\lambda_{1}=\frac{\lambda \mu}{n-\mu} .
$$

The proof of this lemma for $n=1$ is given by [4]. For any $n \in N$ we also can apply Jack's lemma [3].

LEMMA 2.2. If $0<\mu<1,0<\lambda \leq 1$ and $Q \in H$ satisfying

$$
Q(z) \prec 1+\frac{\lambda \mu}{n-\mu} z, \quad Q(0)=1, \quad n \in N,
$$

and if $p \in H, p(0)=1$ and satisfies

$$
Q(z) p^{\alpha}(z) \prec 1+\lambda z
$$

where

$$
0<\lambda \leq \frac{(n-\mu) \sin (\pi \alpha / 2)}{\mid \mu+(n-\mu) e^{i \pi \alpha / 2 \mid}}
$$

then $\operatorname{Re}\{p(z)\}>0$ in $U$.

Proof. If in Lemma 1.1 we put $\lambda_{1}=\lambda \mu /(n-\mu)$, then the condition (1.6) is equivalent to

$$
\sin ^{-1} \lambda+\sin ^{-1} \frac{\lambda \mu}{n-\mu} \leq \frac{\alpha \pi}{2} .
$$

This inequality is equivalent to

$$
\sin ^{-1}\left(\lambda \sqrt{1-\frac{\lambda^{2} \mu^{2}}{(n-\mu)^{2}}}+\frac{\lambda \mu}{n-\mu} \sqrt{1-\lambda^{2}}\right) \leq \frac{\alpha \pi}{2},
$$


or to the inequality

$$
\lambda\left[\sqrt{(n-\mu)^{2}-\lambda^{2} \mu^{2}}+\mu \sqrt{1-\lambda^{2}}\right] \leq(n-\mu) \sin \left(\frac{\alpha \pi}{2}\right) .
$$

From there, after some transformations, we get the following equivalent inequality

$$
\begin{aligned}
& \left\{\left[\mu^{2}+(n-\mu)^{2}\right]^{2}-4 \mu^{2}(n-\mu)^{2} \cos ^{2}\left(\frac{\alpha \pi}{2}\right)\right\} \lambda^{4} \\
& \quad-2(n-\mu)^{2}\left[\mu^{2}+(n-\mu)^{2}\right] \sin ^{2}\left(\frac{\alpha \pi}{2}\right) \lambda^{2}+(1-\mu)^{4} \sin ^{4}\left(\frac{\alpha \pi}{2}\right) \geq 0
\end{aligned}
$$

which is equivalent to the condition (2.6).

By Lemma 1.1 we have that $\operatorname{Re}\{p(z)\}>0$ in $U$.

THEOREM 2.3. Let $f \in A_{n}, 0<\mu<1$ and $f$ satisfy the subordination

$$
f^{\prime}(z)\left(\frac{z}{f(z)}\right)^{1+\mu} \prec 1+\lambda z
$$

where

$$
0<\lambda \leq \frac{n-\mu}{\sqrt{\mu^{2}+(n-\mu)^{2}}}
$$

Then $f \in S^{*}$.

Proof. If we put $Q(z)=(z / f(z))^{\mu}\left(=1+q_{n} z^{n}+\cdots\right)$, then after some calculations, we get

$$
Q(z)-\frac{1}{\mu} z Q^{\prime}(z)=f^{\prime}(z)\left(\frac{z}{f(z)}\right)^{1+\mu} \prec 1+\lambda z .
$$

From Lemma 2.1 we have

$$
Q(z) \prec 1+\lambda_{1} z, \quad \lambda_{1}=\frac{\lambda \mu}{n-\mu} .
$$

The rest part of the proof is the same as in the case $n=1$ (see [4, Theorem 1]) and we omit the details.

THEOREM 2.4. Let $0<\mu<1$ and $0<\alpha \leq 1$. If $f \in A_{n}$ satisfies

$$
\left|f^{\prime}(z)\left(\frac{z}{f(z)}\right)^{1+\mu}-1\right|<\frac{(n-\mu) \sin (\pi \alpha / 2)}{\left|\mu+(n-\mu) e^{i \pi \alpha / 2}\right|}, \quad z \in U
$$

then $f \in S(\alpha)$.

Proof. If we put $\lambda=(n-\mu) \sin (\pi \alpha / 2) /\left|\mu+(n-\mu) e^{i \pi \alpha / 2}\right|$, then, since $0<\alpha \leq$ 1, we have $0<\lambda \leq(n-\mu) / \sqrt{\mu^{2}+(n-\mu)^{2}}$, and by Theorem 2.3, $f \in S^{*}$. Later, the function $Q(z)=(z / f(z))^{\mu}=1+q_{n} z^{n}+\cdots$ is analytic in $U$ and satisfies $Q(z) \prec$ $1+\lambda_{1} z, \lambda_{1}=\lambda \mu /(n-\mu)$. Now, if we define

$$
p(z)=\left(\frac{z f^{\prime}(z)}{f(z)}\right)^{1 / \alpha},
$$


then $p$ is analytic in $U, p(0)=1$ and condition (2.15) is equivalent to

$$
Q(z) p^{\alpha}(z) \prec 1+\lambda z .
$$

Finally, from Lemma 2.2 we obtain

$$
\left(\frac{z f^{\prime}(z)}{f(z)}\right)^{1 / \alpha} \prec \frac{1+z}{1-z}\left(\Longleftrightarrow \frac{z f^{\prime}(z)}{f(z)} \prec\left(\frac{1+z}{1-z}\right)^{\alpha}\right),
$$

that is, $f \in S(\alpha)$.

We note that for $\alpha=1$ we have the statement of Theorem 2.3.

For $n=1, \mu=1 / 2, \alpha=2 / 3$ we get the following corollary.

COROLLARY 2.5. Let $f \in A$ and let

$$
\left|f^{\prime}(z)\left(\frac{z}{f(z)}\right)^{3 / 2}-1\right|<\frac{1}{2}, \quad z \in U .
$$

Then

$$
\left|\arg \left(\frac{z f^{\prime}(z)}{f(z)}\right)\right|<\frac{\pi}{3}, \quad z \in U
$$

that is, $f \in S(2 / 3)$.

THEOREM 2.6. Let $0<\mu<1, \operatorname{Re}\{c\}>-\mu$, and $0<\alpha \leq 1$. If $f \in A_{n}$ satisfies

$$
\left|f^{\prime}(z)\left(\frac{z}{f(z)}\right)^{1+\mu}-1\right|<\left|\frac{n+c-\mu}{c-\mu}\right| \frac{(n-\mu) \sin (\pi \alpha / 2)}{\left|\mu+(n-\mu) e^{i \pi \alpha / 2}\right|}, \quad z \in U,
$$

then the function

$$
F(z)=z\left[\frac{c-\mu}{z^{c-\mu}} \int_{0}^{z}\left(\frac{t}{f(t)}\right)^{\mu} t^{c-\mu-1} d t\right]^{-1 / \mu}
$$

belongs to $S(\alpha)$.

Proof. If we put that $\lambda$ is equal to the right-hand side of the inequality (2.21) and

$$
Q(z)=F^{\prime}(z)\left(\frac{z}{F(z)}\right)^{1+\mu}\left(=1+q_{n} z^{n}+\cdots\right),
$$

then from (2.21) and (2.22) we obtain

$$
Q(z)+\frac{1}{c-\mu} z Q^{\prime}(z)=f^{\prime}(z)\left(\frac{z}{f(z)}\right)^{1+\mu} \prec 1+\lambda z .
$$

Hence, by using the result of Hallenbeck and Ruscheweyh [2, Theorem 1] we have that

$$
Q(z) \prec 1+\lambda_{1} z, \quad \lambda_{1}=\frac{|(c-\mu)| \lambda}{|n+c-\mu|}=\frac{(n-\mu) \sin (\pi \alpha / 2)}{\mid \mu+(n-\mu) e^{i \pi \alpha / 2 \mid}},
$$

and the desired result easily follows from Theorem 2.4. 
REMARK 2.7. For $\alpha=1$ and $n=1$ we have the corresponding result given earlier in [4]. For $c=\mu+1$, we have

COROLLARY 2.8. Let $0<\mu<1$ and $0<\alpha \leq 1$. If $f \in A_{n}$ satisfies the condition

$$
\left|f^{\prime}(z)\left(\frac{z}{f(z)}\right)^{1+\mu}-1\right|<\frac{n(n-\mu) \sin (\pi \alpha / 2)}{\left|\mu+(n-\mu) e^{i \pi \alpha / 2}\right|}, \quad z \in U,
$$

then the function

$$
F(z)=z\left[\frac{1}{z} \int_{0}^{z}\left(\frac{t}{f(t)}\right)^{\mu} d t\right]^{-1 / \mu}
$$

belongs to $S(\alpha)$.

ACKNOWLEDGEMENT. The work of the first author was supported by Grant No. 04M03 of MNTRS through Math. Institute SANU.

\section{REFERENCES}

[1] P. L. Duren, Univalent Functions, Grundlehren der Mathematischen Wissenschaften [Fundamental Principles of Mathematical Science], vol. 259, Springer-Verlag, New York, Berlin, 1983. MR 85j:30034. Zbl 514.30001.

[2] D. J. Hallenbeck and S. Ruscheweyh, Subordination by convex functions, Proc. Amer. Math. Soc. 52 (1975), 191-195. MR 51\#10603. Zbl 311.30010.

[3] I. S. Jack, Functions starlike and convex of order $\alpha$, J. London Math. Soc. (2) 3 (1971), 469474. MR 43\#7611. Zbl 224.30026.

[4] M. Obradović, A class of univalent functions, Hokkaido Math. J. 27 (1998), no. 2, 329-335. CMP 1638 004. Zbl 908.30009.

[5] S. Ponnusamy and V. Singh, Criteria for strongly starlike functions, Complex Variables Theory Appl. 34 (1997), no. 3, 267-291. MR 98j:30010. Zbl 892.30005.

Milutin Obradović: Department of Mathematics, Faculty of Technology and MetALLURGY, 4 KARNEGIJOVA STREET, 11000 BELGRADE, YUGOSLAVIA

E-mail address: obrad@c7ab.tmf.bg.ac.yu

Shigeyoshi OWa: Department of Mathematics, Kinki University, Higashi-OSAKa, OsAKA 577-8502, JAPAN

E-mail address: owa@math.kindai .ac.jp 


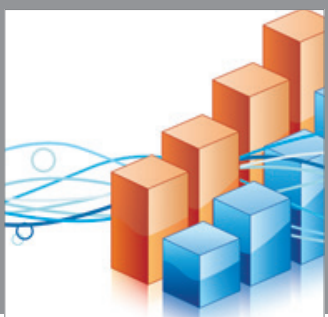

Advances in

Operations Research

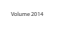

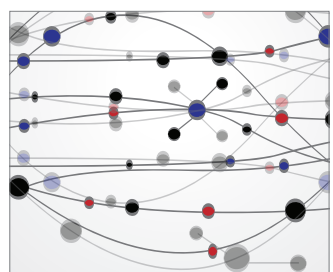

\section{The Scientific} World Journal
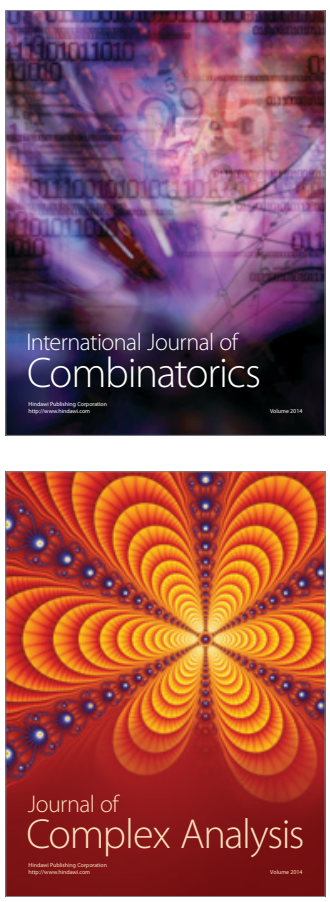

International Journal of

Mathematics and

Mathematical

Sciences
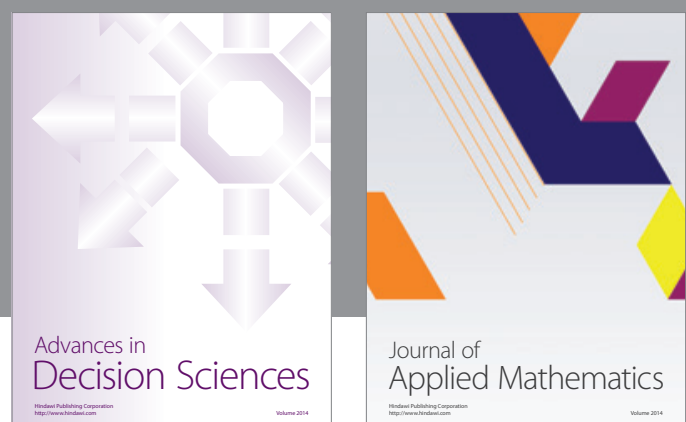

Journal of

Applied Mathematics
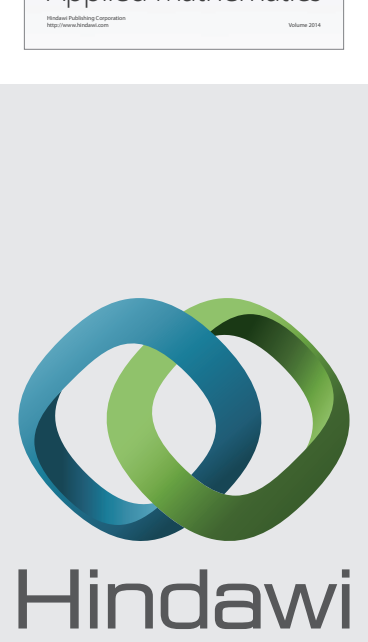

Submit your manuscripts at http://www.hindawi.com
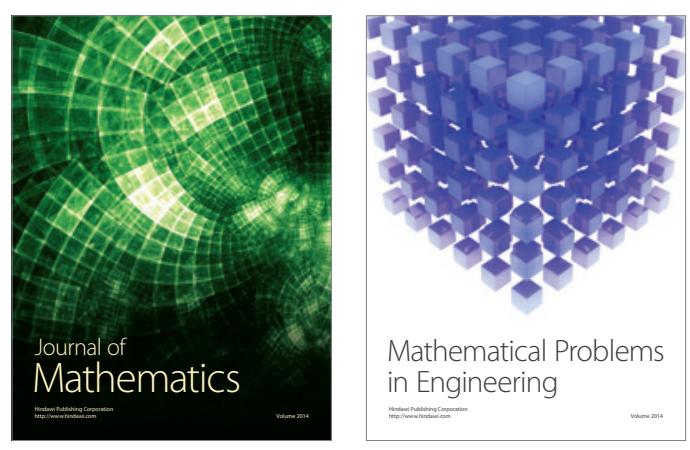

Mathematical Problems in Engineering
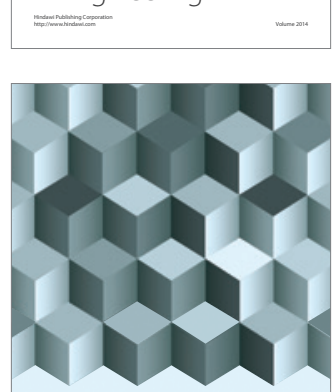

Journal of

Function Spaces
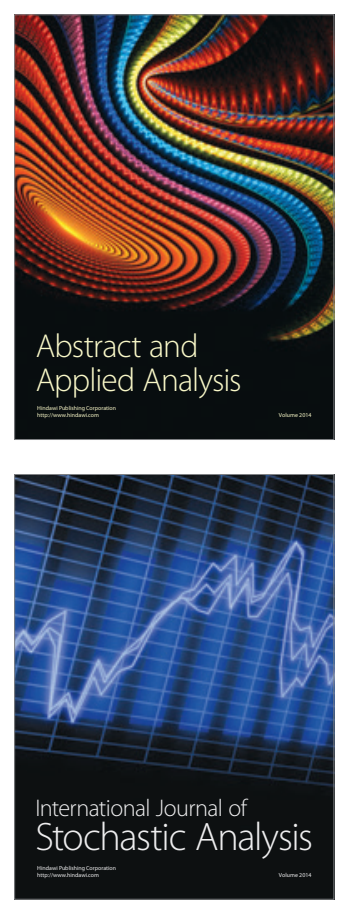

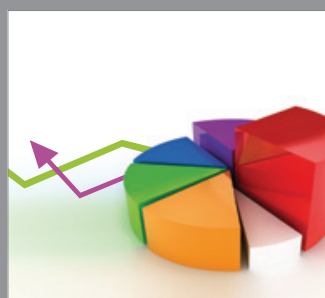

ournal of

Probability and Statistics

Promensencen
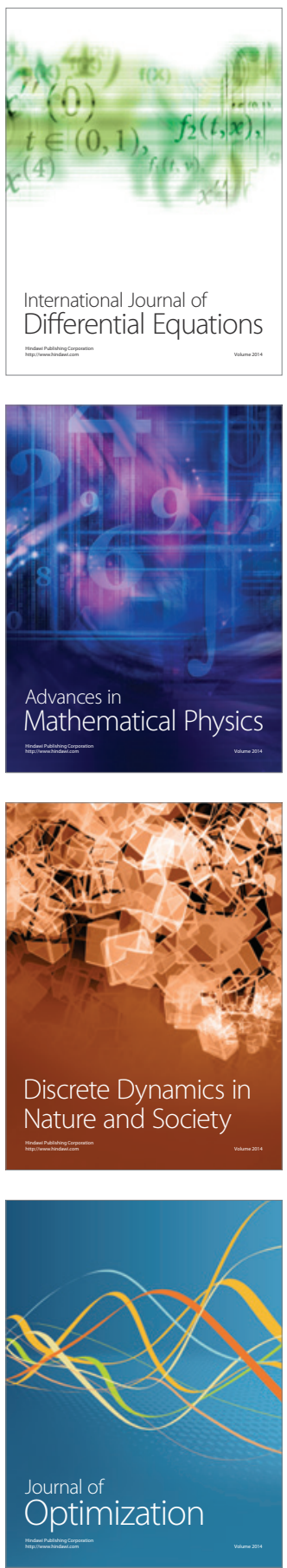